\title{
The Inherent Flexibility of Receptor Binding Domains in SARS-CoV-2 Spike Protein
}

\section{Author Information}

Hisham M. Dokainish ${ }^{\mathrm{a}}$, Suyong Re ${ }^{\mathrm{b}, \mathrm{c}}$, Takaharu Moria ${ }^{\mathrm{a}}$, Chigusa Kobayashi ${ }^{\mathrm{d}}$, Jaewoon Jung ${ }^{\mathrm{d}}$, and Yuji Sugita ${ }^{a, b, d}$

${ }^{\text {a}}$ Theoretical Molecular Science Laboratory, RIKEN Cluster for Pioneering Research, Hirosawa 2-1, Wako, Saitama 351-0198, Japan.

${ }^{\mathrm{b}}$ Laboratory for Biomolecular Function Simulation, RIKEN Center for Biosystems Dynamics Research, 6-71 Minatojima-minamimachi, Chuo-ku, Kobe, Hyogo 650-0047, Japan.

${ }^{\mathrm{c} A r t i f i c i a l ~ I n t e l l i g e n c e ~ C e n t e r ~ f o r ~ H e a l t h ~ a n d ~ B i o m e d i c a l ~ R e s e a r c h, ~ N a t i o n a l ~ I n s t i t u t e s ~ o f ~ B i o m e d i c a l ~}$ Innovation, Health, and Nutrition, 7-6-8, Saito-Asagi, Ibaraki, Osaka 567-0085, Japan.

${ }^{\mathrm{d} C}$ Computational Biophysics Research Team, RIKEN Center for Computational Science, 6-7-1 Minatojimaminamimachi, Chuo-ku, Kobe, Hyogo 650-0047, Japan.

\section{Contributions}

H.M.D. performed simulations. H.M.D., S.R., C.K., T.M. analyzed data and made figures. H.M.D. modelled simulation systems. J.J. and C.K. made software. H..M.D., S.R. and Y.S. wrote manuscript. H.M.D. and Y.S. designed and initiated the research.

\section{Corresponding author}

Corresponding to: Yuji Sugita (sugita@riken.jp). 


\title{
ORCID
}

Hisham M. Dokainish (https://orcid.org/0000-0002-4387-4790)

Suyong Re (https://orcid.org/0000-0002-3752-6554)

Takaharu Mori (https://orcid.org/0000-0002-8717-2926)

Chigusa Kobayashi (https://orcid.org/0000-0002-5603-4619)

Jaewoon Jung (https://orcid.org/0000-0002-2285-4432)

Yuji Sugita (https://orcid.org/0000-0001-9738-9216)

\begin{abstract}
Spike (S) protein is the primary antigenic target for neutralization and vaccine development for the severe acute respiratory syndrome coronavirus 2 (SARS-CoV-2). It decorates the virus surface and undergoes large conformational changes of its receptor binding domain (RBD) to enter the host cell, as the abundant structural studies suggest. Here, we observe Down, one-Up, one-Open, and two-Up-like structures in enhanced molecular dynamics simulations without pre-defined reaction coordinates. The $\mathrm{RBD}_{\mathrm{A}}$ transition from Down to one-Up is supported by transient salt-bridges between $\mathrm{RBD}_{\mathrm{A}}$ and $\mathrm{RBD}_{\mathrm{C}}$ and by the glycan at $\mathrm{N} 343_{\mathrm{B}}$. Reduced interactions between $\mathrm{RBD}_{\mathrm{A}}$ and $\mathrm{RBD}_{\mathrm{B}}$ induce the $\mathrm{RBD}_{\mathrm{B}}$ motions toward two-Up. Glycan shielding for neutralizing antibodies is the weakest in one-Open. Cryptic pockets are revealed at the RBD interfaces in intermediate structures between Down and one-Up. The inherent flexibility in S-protein is, thus, essential for the structure transition and shall be considered for antiviral drug rational design or vaccine development.
\end{abstract}




\section{Introduction}

The severe acute respiratory syndrome coronavirus 2 (SARS-CoV-2) has caused over a 190 million infections and 4 million deaths, as of July 2021 (https://coronavirus.jhu.edu/map.html). It represents an urgent need for an effective medical intervention strategy, to avoid further social and economic consequences ${ }^{1}$. Different types of vaccines, for example, those from Pfizer-BioNTech or Moderna using the mRNA of Spike (S) protein, are currently available, and there are several FDA approved drug candidates under consideration ${ }^{2,3}$. At the same time, more infectious mutants such as B.1.617.2 (Delta) and B.1.427 / B.1.429 (Epsilon) have appeared, and some evade from the immune system ${ }^{4,5,6,7}$. Furthermore, the virus ability to infect a wide range of vertebrates, which could act as a reservoir, points out the future risk despite the vaccination progress ${ }^{8}$. A deeper understanding of the virus molecular structure and infection mechanism is crucial to stop the virus transmission including mutant strains 9 .

SARS-CoV-2, an enveloped positive single stranded RNA (+ssRNA) virus, has a large genome of approximately $30 \mathrm{~kb}$ encoding 29 proteins $^{9,10}$. A transmembrane homotrimeric class I fusion glycoprotein decorating the virus, known as S-protein, plays a critical role in the viral cell entry ${ }^{11,12}$. In an immediate response to the pandemic, over a hundred Cryo-electron microscopy (EM) and X-ray crystal structures of Sprotein have been reported and rapidly advanced in our understanding of the S-protein/receptor binding mechanism ${ }^{13,14,15,16,17,18,19}$. The N-terminal subunit (S1), which is composed of the N-terminal domain (NTD), the receptor binding domain (RBD) and two other subdomains (SD1 and SD2) (Figure S1) ${ }^{16,20}$, initially binds to the host cell receptor angiotensin-converting enzyme $2(\mathrm{ACE} 2)^{19}$. This binding is followed by priming of the C-terminal subunit (S2) and its large conformational change leading to the membrane fusion for the cell entry $^{12,21}$. S-protein is covered by $66 \mathrm{~N}$-glycans, 22 per protomer, to evade from the host cell immune system $^{22,23}$. To block the initial binding with ACE2 either by stimulating the immune system using vaccines or by neutralized antibodies or small-molecule drugs is the primary target for medical interventions. Numerous Cryo-EM structures reveal that the RBDs can take inactive Down and active Up forms including one-Up, twoUp and three-Up conformations (Fig. 1a) $)^{13,14,15,16,17,21,24,25}$. The RBDs are considered to undergo large conformational changes from the receptor inaccessible Down to the receptor accessible Up states in the ACE2 binding ${ }^{16,19,26}$. Note that most of the Cryo-EM structures representing the active Up forms were determined together with other proteins, such as antibodies or a fragment of ACE2 (Table S1). A recent single molecule 
fluorescence resonance energy transfer (smFRET) experiment suggested that the Down-to-Up transition occurs in S-protein even without its ligand, taking at least one transient intermediate ${ }^{27}$. This suggests the necessity to explore a wide conformational space of S-protein for describing transition pathways and intermediate structures. It would give us better understandings of the virus entry mechanisms and contribute to the development of antiviral drugs or antibodies.

Molecular dynamics (MD) simulations at the atomic level have been conducted to explore the conformational stability of S-protein by including the surface glycans, which are largely missed in the experimental structures. Several microsecond-scale MD simulations showed conformational flexibility of the stalk in S-protein, different levels of glycan-shielding between Down and Up, and their relevance in the ACE2 binding ${ }^{22,28,29}$. They also identified specific interactions between side-chain residues or those with glycans to stabilize either Down or $\mathrm{Up}^{30,31}$. The timescale of the Down-to-Up transitions in S-protein is far slower than that attainable one in the current MD simulations, leaving the inherent flexibility and transition pathways largely unknown. To overcome such difficulties, several enhanced sampling methods have been applied to investigate the Downto-1Up-transition, including targeted $\mathrm{MD}^{31}$, steering $\mathrm{MD}^{32}$, nudged elastic band/umbrella sampling ${ }^{33}$, adaptive sampling, ${ }^{34}$ and weighted ensemble methods ${ }^{35}$. In most of these simulations, pre-defined reaction coordinates and/or bias potentials along the coordinates are used to enhance the motions of a single RBD in S-protein.

Here we apply the generalized replica exchange with solute tempering of selected surface charged residues $(\text { gREST_SSCR })^{36}$, which enhances domain motions of a protein by exchanging the solute temperature of selected surface residues.gREST_SSCR is distinct from the other simulations applied to S-protein in that no reaction coordinates as well as bias potentials are used, allowing us to examine the inherent flexibility of Sprotein involving more than one RBD. As listed in Table 1, the RBD motion in the RBD/SD1 monomer, the trimeric S-protein in the presence and absence of glycans, is systematically investigated. The intrinsic flexibility of S-protein observed in the enhanced sampling MD simulations suggests new mechanisms underlying the Down-to-Up transitions, glycan shielding for the binding with antibodies, and unprecedented cryptic pockets in intermediate structures during the transitions. 


\section{Results}

\section{Enhanced RBD motions in gREST_SSCR simulations}

Table 1 lists all simulations performed in this study, wherein three gREST_SSCR simulations are involved. Two starting with a Down Cryo-EM structure (PDBID: 6VXX ${ }^{20}$ ) in the presence (gREST_Down) and absence (gREST_Down w/o glycan) of glycans and one from one-Up structure (PDBID: 6VYB ${ }^{20}$ ) (gREST_Up). All were solvated and ionized after modeling missing residues (SI Methods, Figures. S1 and S2). The simulations were performed using GENESIS software $(2.0 \text { beta })^{37}$, which was designed to achieve high performance and scalability on the supercomputer Fukagu ${ }^{38}$. In the solvated S-protein system including about 655,000 atoms, 2,048 nodes on Fugaku were used to achieve the performance of around $52 \mathrm{~ns} /$ day. The total simulation times including all the replicas are $8 \mu s, 4.8 \mu \mathrm{s}$ and $2.4 \mu \mathrm{s}$ in gREST_Down, gREST_Up and gREST_Down w/o glycan, respectively.

Table 1. MD simulations of S-protein performed in this study.

\begin{tabular}{|l|l|l|l|}
\hline Name & Model & Method & Simulations Length \\
\hline gREST_Down & Spike Down w/glycans & gREST_SSCR & $500 \mathrm{~ns} \times 16$ replicas \\
\hline gREST_Up & Spike Up w/ glycans & gREST_SSCR & $300 \mathrm{~ns} \times 16$ replicas \\
\hline gREST_Down w/o glycan & Spike Down w/o glycans & gREST_SSCR & $150 \mathrm{~ns} \times 16$ replicas \\
\hline Monomer_Down & RBD/SD1 monomer Down & cMD & $300 \mathrm{~ns} \times 1$ run \\
\hline Monomer_Up & RBD/SD1 monomer Up & cMD & $300 \mathrm{~ns} \times 2$ runs \\
\hline cMD_Down & & & $1,000 \mathrm{~ns} \times 1$ run \\
\hline cMD_Up & Spike Down w/ glycans & cMD & $1,000 \mathrm{~ns} \times 1$ run \\
\hline
\end{tabular}

${ }^{*}$ Classical MD simulations of S-protein performed in our previous study ${ }^{31}$.

gREST_SSCR facilitates conformational sampling of a multi-domain protein by scaling the Coulomb and Lennard-Jones interactions of selected charged residues at domain interfaces through the solute temperature exchanges $^{36}$. Here, 8 pairs of positively and negatively charged residues ( 870 atoms in total) in the RBD region 
were selected as the solute region of gREST_SSCR (Figure S3a). 16 copies of each simulation system were used to cover the solute temperature range from 310 to $545 \mathrm{~K}$. For this method to work, the solute temperatures between neighboring replicas must be exchanged frequently. This, indeed, happened in all three simulations, showing sufficient overlaps of the potential energy distributions between neighboring replicas (Figure S3). All simulations show reasonable solute temperature exchange with the average acceptance ratio of $21.2 \%, 20.3 \%$ and 20.8\% for gREST_Down, gREST_Up, and gREST_Down w/o glycan, respectively. The SD1/RBD hinge angle and the $\mathrm{C} \alpha$ root mean square deviation (RMSD) of each RBD upon fitting S2 provide simple measures of the Down-to-Up transitions: the RMSD and hinge angle in Up take the values of about $20 \AA$ and $150^{\circ}$, respectively, while the hinge angle in Down is about $116^{\circ}$. Since gREST_SSCR is free from any pre-defined reaction coordinates, the Down-to-Up transitions happened not only in $\mathrm{RBD}_{\mathrm{A}}$ but also in $\mathrm{RBD}_{\mathrm{B}}$ or $\mathrm{RBD}_{\mathrm{C}}$. Even two-Up-like conformations were observed in some replicas within $500 \mathrm{~ns} /$ replica (for instance, replica 8 in gREST_Down, replica 4 in gREST_Up, and replica 16 in gREST_Down w/o glycan) (Figure S4). Despite the large-scale motions, intra-domain structures of three RBDs and NTDs were kept stable. The C $\alpha$ RMSD of RBD and NTD upon fitting to their own structures at $310 \mathrm{~K}$ in gREST_Down reveal about 1.4 and $2.2 \AA$, respectively (Figures S5a and S5b). They are comparable to those in the conventional $\mathrm{MD}^{31}$ (1.4 and $1.6 \AA$ for RBD and NTD). Slightly larger RMSD values of NTD in gREST_SSCR are attributed to the loop regions abundant in NTD, as indicated in root mean square fluctuations (RMSF) (Figure S5c and S5d).

\section{Comparison between Cryo-EM and simulated structural ensembles}

As of December 2020, 126 Cryo-EM structures of S-protein, including all Down, one-Up, two-Up and threeUp conformations, were deposited in the Protein Data Bank (Table S1). To describe the Down-to-Up transitions found in the Cryo-EM structures, we introduced three computational techniques: (i) the 9-beads representation per protomer as used in the studies by Henderson et al. ${ }^{7,39}$ to build up a 27-beads model of Sprotein (Figure 1a and Table S2), (ii) the rotation scheme to make the most significant RBD motion always happen in Chain ${ }_{\mathrm{A}}$ (Figures S6 and S7), and (iii) the principal component analysis (PCA) on the 27-beads model of S-protein upon fitting all the beads to reduce the essential dimensions in the conformational space. In Figure 1b, the first principal component (PC1) represents a symmetric Down-to-Up motion involving all three RBDs, while the second component (PC2) reveals an asymmetric motion of RBDs where only $\mathrm{RBD}_{\mathrm{A}}$ undergoes the 
Down-to-Up motion. The two lowest PCs cover about $85 \%$ of the conformational fluctuations observed in the Cryo-EM structures. In Figure 1c, S-protein Cryo-EM structures with distinct RBD conformations, Down, one-Up, two-Up, and three-Up, are found in different clusters on the PC1-PC2 space (Figure 1c).

Next, we project the results of our previous $1 \mu$ s conventional MD simulations (cMD_Down and cMD_Up) and the three gREST_SSCR simulations on the same space (Figure 1c). To obtain the distributions at $310 \mathrm{~K}$ in gREST_SSCR, we applied Multistate Bennett Acceptance Ratio (MBAR) ${ }^{40}$ for utilizing all the trajectories at different solute temperatures. The distributions of cMD_Down and cMD_Up overlap with the corresponding Cryo-EM structures (Down and one-Up, respectively), while there is a big gap between the two simulations. Instead, the distributions of gREST_SSCR simulations reach from the starting state to another: gREST_Down: from Down to one-Up, gREST_Up: from one-Up to two-Up, gREST_Down w/o glycan: from Down to oneUp. Due to the insufficient computational times, the initial structure dependences on distributions are found in all the three gREST_SSCR simulations. This issue would be resolved if we continue the simulations much longer timescales. More importantly, gREST_SSCR could cover more than one state, opening the possibility to investigate inherent flexibility of S-protein, transition pathways, and the intermediate structures. The distribution of gREST_Down w/o glycan is wider than that in gREST_Down, suggesting that glycans on the surface of S-protein play key roles in regulating RBD motions.

To focus on the Down-to-Up motions in S-protein, we define the hinge and twist angles using the C $\alpha$ atoms in RBD and SD1 (Figure 2b). The hinge angle directly describes the Down-to-Up transitions, while the twist angle explains side motions of RBD. Larger values of the hinge and twist angles reveal the transition toward Up conformations. The gREST_SSCR distributions at $310 \mathrm{~K}$ on the hinge-twist angle space are compared to protomers of the 126 Cryo-EM structures in Figure S8, showing a good overlap between the Cryo-EM structures and structure ensembles at $310 \mathrm{~K}$ in gREST_SSCR as we see on the PC1-PC2 space. Interestingly, Monomer_Up, which consists of only a monomer containing SD1 and RBD (Up) sampled Down, one-Up, and one-Open states within $300 \mathrm{~ns}$ cMD simulations. The simulated ensembles in Monomer_Up is close to the Cryo-EM structures and the gREST simulations containing a whole S-protein.

On the Hinge ${ }_{\mathrm{A}}-\mathrm{Hinge}_{\mathrm{B}}$ space at $310 \mathrm{~K}$, the Down-to-Up motion is also identified in Chain $\mathrm{B}_{\mathrm{B}}$ in gREST_Up simulations starting from the one-Up structure in $\mathrm{RBD}_{\mathrm{A}}$ (Figure $2 \mathrm{c}$ ). We did not observe similar motions in the Hinge $\mathrm{A}_{\mathrm{A}}-\mathrm{Hinge}_{\mathrm{C}}$ space, suggesting that the Up motions of $\mathrm{RBD}_{\mathrm{B}}$ is followed from $\mathrm{RBD}_{\mathrm{A}}$ to form two-Up 
structures. Taking together the information on the PC1-PC2 and the Hinge $\mathrm{A}_{\mathrm{A}}-\mathrm{Hinge}_{\mathrm{B}}$ spaces, four major structures were identified: the Down symmetric $\left(\mathrm{D}_{\mathrm{Sym}}:\left(\operatorname{Hinge}_{\mathrm{A}}, \mathrm{Hinge}_{\mathrm{B}}\right)=\left(114.9^{\circ}, 112.5^{\circ}\right)\right)$, one-Up $(1 \mathrm{U}$ : $\left.\left(158.3^{\circ}, 112.7^{\circ}\right)\right)$, one-Up-open $\left(1 \mathrm{U}_{\mathrm{O}}:\left(163.3^{\circ}, 108.9^{\circ}\right)\right)$, and two-Up-like $\left(2 \mathrm{U}_{\mathrm{L}}:\left(150.6^{\circ}, 148.6^{\circ}\right)\right)$. They are superimposed to the Cryo-EM structures having corresponding RBD conformations in Figure 2d. The simulation-derived $\mathrm{D}_{\mathrm{Sym}}$ and $1 \mathrm{U}$ conformations are better aligned to the recent high-resolution structures of Down (PDB:6ZGE) $)^{13}$ and one-Up (PDB:6XKL) ${ }^{14}$, respectively. Intriguingly, $\mathrm{RBD}_{\mathrm{A}}$ in $1 \mathrm{U}_{\mathrm{O}}$ is well aligned to that in one of the RBDs in three-UP Cryo-EM structure (PDB:7DCC) $)^{41} \cdot \mathrm{RBD}_{\mathrm{A}}$ and $\mathrm{RBD}_{\mathrm{B}}$ in $2 \mathrm{U}_{\mathrm{L}}$ also agree well with those in two-Up Cryo-EM structure (PDB:6X2B) ${ }^{39}$, although $2 \mathrm{U}_{\mathrm{L}}$ in gREST_SSCR remains interdomain interactions between $\mathrm{RBD}_{\mathrm{A}}$ and $\mathrm{RBD}_{\mathrm{B}}$, which is completely lost in the two-Up Cryo-EM structures.

\section{The accessibility of RBD in different conformations}

To get insights into the contribution of each conformation to the ACE2 and neutralizing antibodies (nAbs) bindings, the accessibility of RBD is examined in terms of the Solvent Accessible Surface Area (SASA) ${ }^{30}$. Figures $3 \mathrm{a}$ and $3 \mathrm{~b}$ (and Figures S9-S11) show the per-residue SASA around the receptor binding motif (residues $\mathrm{I} 410$ to V510, referred to as RBM hereafter) calculated for $\mathrm{D}_{\text {Sym }}, 1 \mathrm{U}, 1 \mathrm{U}_{\mathrm{O}}$ and $2 \mathrm{U}_{\mathrm{L}}$ conformations. Four mutational residues of concern, K417, L452, E484, and N5015 , are highlighted in red. In Down conformation ( $D_{\text {Sym }}$ ), glycans at N165 and N343 largely shield RBM, leaving only small part accessible. RBM SASA increases in one-Up conformations ( $1 \mathrm{U}$ and $1 \mathrm{U}_{\mathrm{O}}$ ) compared to Down, wherein $1 \mathrm{U}_{\mathrm{O}}$ exhibits the utmost increase suggesting its potential contribution to the receptor and/or antibody binding. In contrast, the SASA increases only slightly, even decreases locally around residue C480, in two-Up $\left(2 U_{L}\right)$ conformation due to the mutual interactions between two RBDs. This finding suggests that one-Up conformations are the primary target of nAbs and the bindings of multiple nAbs likely proceed in a stepwise manner through one-Up conformations. Notably, K417 and N501 are accessible only in one-Up conformations as expected, while E484 is accessible in both Down and Up, inferring a widespread effect of E484 mutations. On the other hand, L452 is not accessible in any conformations and hence is expected to rarely affect the binding with either receptor or antibody, in wild type. ${ }^{42}$ In contrast L452R mutation has shown to reduce neutralization ${ }^{5}$, suggesting its indirect contribution (e.g. the alternation of the S-protein conformational equilibria) to the immune escape. 
Figure $3 \mathrm{c}$ shows the calculated SASA for each epitope region of the four classes of nAbs (Class I: binds to Up for blocking ACE2, Class II: binds to Up and Down for blocking ACE2, Class III: binds outside RBM but recognize Up and Down, Class IV: binds Up without blocking ACE2) ${ }^{5}$. The epitope regions of Class I and II antibodies are largely exposed and almost completely de-shieled from glycans in Up conformations. On the other hand, the epitope region for Class III antibody is less exposed and largely shielded by glycans. This agrees with that Class III antibodies recognize "glycoepitopes"43. Note that the epitope region of Class IV antibody is slightly exposed only in $1 \mathrm{U}_{\mathrm{O}}$ conformation. Further opening of RBD may allow the binding of Class IV antibody, such as CR3022, as suggested in a previous computational study ${ }^{34}$. Figure $3 \mathrm{c}$ shows the putative binding models with antibodies obtained by aligning the antibodies in Cryo-EM structures with each of Up conformations (see Figure S12 and S13 for detail). The 1U conformations can be targeted by any of Class I-III antibodies. On the other hand, $1 \mathrm{U}_{\mathrm{O}}$ and $2 \mathrm{U}_{\mathrm{L}}$ conformations are likely bound with Class I/II and Class II/III, respectively, suggesting the possible evade from some antibody attacks.

\section{Comparison between smFRET and simulated structural ensembles}

Using smFRET, Lu et al. showed the conformational dynamics of S-protein in the presence or absence of its receptor, hACE2. ${ }^{27}$ They characterized four structural ensembles including two types of Down (major and minor), one intermediate and one-Up, suggesting the inherent flexibility of the RBD region regardless of the receptor binding. To examine whether gREST_SSCR could reproduce the experimental data, we first classified each gREST_SSCR trajectory at $310 \mathrm{~K}$ by means of $k$-means clustering and re-clustering guided by the hinge and twist angles distributions (Figures S14-S16, Table S3) and then computed the distance between residues 425-431 in $\mathrm{RBD}_{\mathrm{A}}$ and residues 554-561 in $\mathrm{SD} 1_{\mathrm{A}}$ to correlate the smFRET intensity by Lu et al. ${ }^{27}$ By combining the distance distributions from gREST_Down and gREST_Up, we obtained the five conformational ensembles (Figure S17): Down symmetric (Down ${ }_{\text {Sym }}$ ), Down like (Down Like , two intermediates (Int2 and Int3), and Up (1Up). The smFRET distance alone cannot distinguish various $\mathrm{Up}$ conformations including $1 \mathrm{U}, 1 \mathrm{U}_{\mathrm{O}}$, and $2 \mathrm{U}_{\mathrm{L}}$ and thus we refer them to as $1 \mathrm{Up}$. Down ${ }_{\text {Sym }}$ and Down $n_{\text {Like }}$ give distributions in the range of 30-35 $\AA$, while $1 \mathrm{Up}$ shows around a median distance of $47 \AA$. The distributions of Int 2 and Int 3 are median distances of around 38 $\AA$ and $40 \AA$, respectively. Since Int 3 has a large distance distribution that overlaps with Int 2 and both intermediates might be indistinguishable in the smFRET experiment, the four conformations corresponding to 
the smFRET intensity of $0.8,0.5,0.3$ and 0.1 in the absence of hACE2 ${ }^{27}$ might be Down ${ }_{\text {Sym, }}$ Down $n_{\text {Like, }}$ Intermediate (Int2 and Int3) and 1Up. Each ensemble is also characterized with hinge angle distributions: Down $\mathrm{Sym}_{\text {Sy }}$ Hinge $<120^{\circ}$, Down $_{\text {Like }}$ : Hinge $<130^{\circ}$, Int2:120 $<$ Hinge $<140^{\circ}$, Int3:Hinge $\sim 140^{\circ}, 1 \mathrm{Up}: 140^{\circ}<$ Hinge $<160^{\circ}$.

\section{Transition pathways and transient interactions stabilizing the intermediate}

\section{structures}

Based on the free-energy landscapes at $310 \mathrm{~K}$ and the comparison between the gREST simulations and smFRET experiments, we now have evidence on the inherent flexibility of S-protein in the absence of its ligand. We next focus on molecular mechanisms underlying the Down-to-1Up transitions in S-protein in terms of the correlated motions of the hinge and twist angles in three RBDs (Figure S18). Note that such correlated motions are hardly obtained using targeted MD simulations (Figure S19) or the MD simulations enhanced with pre-defined reaction coordinates ${ }^{35,44}$. As expected, Hinge $\mathrm{A}_{\mathrm{A}}$ and $\mathrm{Twist}_{\mathrm{A}}$ are highly correlated with each other (Figure S18c), while Hinge ${ }_{\mathrm{A}}$ and Hinge $\mathrm{B}_{\mathrm{B}}$ show almost no correlations (Figure S18a). Some correlations exist between Hinge $\AA$ and $\mathrm{Hing}_{\mathrm{C}} /$ Twist $_{\mathrm{B}}$, which appear to relate with the preference of two intermediates.

To examine the origin of such correlated motions in three RBDs, contact analysis (Figure S20) and hydrogen bond (HB) analysis (Figure S21) were carried out for each cluster observed in gREST_Down and gREST_Up simulations as we did in the previous study ${ }^{31}$. In Figure 4, several key contacts and hydrogen bonds are highlighted to see drastic changes of the interactions in the Down-to-1Up transition. The glycans at N343 ${ }_{\mathrm{B}}$ and $\mathrm{N} 234_{B}$ switch the interactions with $\mathrm{RBD}_{\mathrm{A}}$ along the transition from Down to one-Up-like. In the intermediates, I2a and I3a, the glycan at $\mathrm{N} 343_{\mathrm{B}}$ changes its contact partners by inserting underneath $\mathrm{RBD}_{\mathrm{A}}$. Concurrently, the formation of salt-bridge interactions between $\mathrm{RBD}_{\mathrm{A}}$ and $\mathrm{RBD} \mathrm{C}_{\mathrm{C}}$, for instance, $\mathrm{R} 408_{\mathrm{A}}-\mathrm{D} 405_{\mathrm{C}}$ and $\mathrm{R} 408_{\mathrm{A}}-\mathrm{D} 406_{\mathrm{C}}$, lift up $\mathrm{RBD}_{\mathrm{A}}$ from its Down to intermediate structures. Finally, in $1 \mathrm{U}_{\mathrm{L}}, \mathrm{N} 234_{\mathrm{B}}$ inserts into the newly formed cavity between $\mathrm{RBD}_{\mathrm{A}}$ and $\mathrm{RBD}_{\mathrm{B}}$, forming new contacts with $\mathrm{D} 428_{\mathrm{A}}$ and $\mathrm{T} 430_{\mathrm{A}}$. The glycan at $\mathrm{N} 165_{\mathrm{B}}$ is in contact with $\mathrm{RBD}_{\mathrm{A}}$ throughout the transition, likely acting as a main barrier for the transition.

We can also characterize the $1 \mathrm{Up}$-to-2Up transition (Figure $\mathrm{S} 22 \mathrm{a}$ ), where $\mathrm{RBD}_{\mathrm{B}}$ undergoes the transitions. The increase in $\mathrm{RBD}_{\mathrm{B}}$ hinge angle is accompanied with a slight decrease in $\mathrm{RBD}_{\mathrm{A}}$ hinge angle. The latter decrease 
likely relates with the increases of the contacts and $\mathrm{HBs}$ between $\mathrm{RBD}_{\mathrm{A}}$ and $\mathrm{RBD}_{\mathrm{C}}$ from $1 \mathrm{Ua}$, the top populated cluster in $1 \mathrm{Up}$, to two-Up-like structures, for instance, $\mathrm{K} 378_{\mathrm{A}}-\mathrm{E} 484_{\mathrm{C}}, \mathrm{Y} 369_{\mathrm{A}}-\mathrm{N} 487_{\mathrm{C}}$ and $\mathrm{D} 427_{\mathrm{A}}-\mathrm{Y} 505_{\mathrm{C}}$ (Figures S20 and S21). The numbers of contacts and HBs in two-Up-like structures are much less than those in one-Up, suggesting that a drastic reduction of inter-domain interactions is required toward a full two-Up conformations. No specific protein-glycan interactions are found to support the $1 \mathrm{Up}$-to-2Up transition.

Finally, we examine the effect of glycans on the Down-to-Up transitions. Figure S22b shows the distributions of gREST_Down w/o glycan simulation on the Hinge $\mathrm{A}_{\mathrm{A}}-\mathrm{Hinge}_{\mathrm{B}}$ space. Without glycans on the surface of Sprotein, the structure distribution becomes wider, suggesting that S-protein is more flexible without glycans. In addition, Down conformations are more diverse and asymmetric as one RBD has larger hinge angle distribution than the others. The transition mechanisms seem to be the same as those with glycans: $\mathrm{RBD}_{\mathrm{A}}$ and $\mathrm{RBD}_{\mathrm{C}}$ form transient interactions to support the Down-to-1Up transition. However, the two-Up-like structure $\left(2 \mathrm{Ub}_{\mathrm{L}}\right)$ is more connected with Down distribution, suggesting that structural integrity of S-protein is lost without glycans on the surface.

\section{Searching for cryptic binding pockets in the intermediate structures}

We applied a machine learning based algorithm (P2Rank ${ }^{45}$ to search for the formation of druggable pockets in the intermediates structures, I2a, I3a and I3b (Figure S23a). The same search was also carried out for Down and one-Up structures for comparison. Figure 5a shows the formation of two cryptic pockets (pocket1 and pocket2) at the interface of RBD in I2a, which are not observed in Down or one-Up. Table S4 lists these predicted pockets showing relatively high scores in all the intermediates. To test the druggability of the two pockets, we performed virtual screening of FDA approved drugs from ZINC database ${ }^{46}$, where we docked 2,115 molecules to the RBD interfaces in I2a, I3a and I3b (following the procedure sketched in Figure 23c). Table S5 shows a list of the index (ID) and binding energies of top ranked molecules. Notably for Nilotinib (Figure 5b), which is ranked at the top 5 and 6 , the previous study has shown that it affects the SARS-Cov-2 infectivity ${ }^{47}$. The authors attributed the effect to the reduction of cell entry, but the mechanism remains unknown ${ }^{47}$. Figures $5 \mathrm{c}$ and S23d show the top-ranked binding poses of Nilotinib in the three intermediates. Nilotinib binds to either pocket1 or pocket 2 in all first 9 binding modes with high binding affinity (Table S6). 
These results serve the two cryptic pockets as potential targets to stabilize intermediate structures and to prevent the formation of one-Up conformation responsible for the viral entry.

\section{Discussion}

\section{Sampling the conformational space of S-protein}

Due to the importance of the conformational changes of S-protein in the infection mechanisms and the rational design of antiviral drugs or antibodies, many extensive simulation studies on S-protein have been carried out since the start of the pandemic. The latest challenges involve the use of a million of distributed computer resources, Folding@home, to realize MD simulation of the SARS-CoV-2 proteome for 0.1 second, in total. An alternative approach is to incorporate experimental observation to effectively sample different states. Brotzakis and co-workers recently applied the Cryo-EM Metainference method to determine the opening pathway and intermediates based on the experimental density map ${ }^{44}$. Yet another approach is to use enhanced sampling methods. Sztain and co-workers performed the weighted ensemble simulations, collecting 130 microseconds trajectories, to characterize the opening pathway ${ }^{35}$. Fallon et al. employed the umbrella sampling, nudged elastic band, and steered MD to characterize the transition pathway and associated cryptic pockets ${ }^{33}$. Our gREST_SSCR simulations aimed to enhance sampling of multi-domain proteins like S-protein by exchanging the solute temperatures of selected surface charged residues. It is free from reaction coordinates and bias potentials, which were used in many of the previous enhanced sampling simulations of S-protein. By using Fugaku supercomputer as well as GENESIS program (ver2.0) designed for achieving high scalability on that system ${ }^{37,38}$, the simulations explored a wide conformational space covering Down, one-Up, one-Open, and two-Up-like structures. The structure ensembles that we obtained in the two gREST_SSCR simulations agree with the Cryo-EM structure distributions and smFRET data, suggesting inherent flexibility of S-protein structures even without its ligand, such as hACE2 or nAbs.

Besides the enhancement of conformational sampling by gREST_SSCR, there are several remaining issues in the simulations. Within our computational time, one single simulation cannot cover a whole free-energy landscape containing all the important structures of S-protein. Three-Up structures, which were found in Cryo$\mathrm{EM}$, for instance, PDB:7DCC ${ }^{41}$, were not observed in our simulations. As we discuss in the next section, 
glycans on the surface of S-protein seem to have dual roles in the transitions, either helping or hindering the Down-to-Up transitions, which makes conformational sampling via MD simulations more difficult. However, conformational sampling via gREST_SSCR is much better than cMD without any pre-defined reaction coordinates and/or bias potentials, providing sufficient structural information to investigate inherent flexibility of S-protein and the transition mechanisms.

\section{Role of Flexible Down structures in the Down-to-Up transitions}

In this study, we observed inherent flexibility of S-protein structures in absence of its ligand, exploring Down, one-Up, one-Open, and two-Up-like structures at $310 \mathrm{~K}$. The trimeric RBDs in S-protein is intrinsically flexible even in Down form, as their interfaces are electrostatically repulsive as shown in our previous study ${ }^{31}$. Not only symmetric structures but also anti-symmetric structures are also observed in Down, suggesting the existence of "flexible Down structure" (Figure S24). This flexibility can initiate the Down-to-1Up transition, by reducing inter-domain interactions found in Down ${ }_{\mathrm{Sym}}$ and allowing the insertion of a glycan at N343 underneath of $\mathrm{RBD}_{\mathrm{A}}$ (Figure $\mathrm{S} 25$ ). This is consistent with the experimentally mobile RBD conformations in Down observed by Ke et al. ${ }^{11}$ and Wrobel et al. ${ }^{13}$.

Following the flexible Down structure, our simulations elucidate molecular mechanisms underlying the Downto-1Up transition involving $\mathrm{RBD}_{\mathrm{A}}$ and the $1 \mathrm{Up}$-to-2 $\mathrm{Up}$ transitions involving $\mathrm{RBD}_{\mathrm{A}}$ and $\mathrm{RBD}_{\mathrm{B}}$. This sequence of events can be explained via the changes of inter-domain interactions as well as protein-glycan interactions. Since $\mathrm{RBD}_{\mathrm{A}}-\mathrm{RBD}_{\mathrm{C}}$ interaction becomes stronger in the Down-to-1Up transition as we see in Figures 4, S20, and $\mathrm{S} 21, \mathrm{RBD}_{\mathrm{B}}$ is more mobile for the next $1 \mathrm{Up}$-to-2Up transition. Among the inter-domain interactions, we pointed out the importance of transient salt-bridges and HBs between $\mathrm{R} 408_{\mathrm{A}}-\mathrm{D} 405_{\mathrm{C}}$ and $\mathrm{R} 408_{\mathrm{A}}-\mathrm{D} 406_{\mathrm{C}}$ for stabilizing the intermediates (Figures 4 and S21). The salt-bridge between R408 ${ }_{\mathrm{A}}$ and D405 $\mathrm{C}$ was also observed in the weighted ensemble simulations reported by Sztain et al. ${ }^{35}$ This scenario is perturbed via protein-glycan interactions, in particular, those involving three glycans at $\mathrm{N} 165_{\mathrm{B}}, \mathrm{N} 234_{\mathrm{B}}$, and $\mathrm{N} 343_{\mathrm{B}}$ as pointed out in previous studies $^{29,30,31}$. In the current study, we highlighted the dual roles of these three glycans: the stabilization of one of the states and the driving force toward the other state. The glycans at N165 stabilize Down, representing a barrier for the Down-to-Up transition, while the glycan at N343 $3_{B}$ drives the transition. Finally, the glycan at N234 Stabilizes one-Up-like conformation. This picture coincides with the experimental results by Henderson 
et $\mathrm{al}^{48}{ }^{48}$, where the population of one-Up drastically is reduced by the glycan deletions at N234 but increases by those at N165. As suggested previously ${ }^{30}$, the glycans at N165 may also stabilize one-Up conformation but as a minor role because it can adapt various orientations to that conformation (Figure S25). The role of glycans at N343 is noteworthy in that it helps the position of RBD lift up from Down and supports it throughout the intermediates. This well explains the 20-fold reduction of the infectivity upon N343Q mutation observed by Li et al. ${ }^{49}$. Recently, a similar role of the glycans at N343 has been proposed by Sztain et al. ${ }^{35}$

\section{Implication for vaccine and drug developments}

Since the drug repurposing studies ${ }^{50,51,52}$ so far mostly target Down and Up conformations from Cryo-EM structures, the identification of druggable cryptic pockets in transient intermediates would introduce unprecedented drug targets ${ }^{44}$. Two cryptic pockets at the RBD interface are identified in the highly populated intermediates along the Down-to-1Up transition. From our virtual screening of FDA approved molecules, these pockets are druggable and accommodates several small molecules including Nilotinib. Intriguingly, Cagno et al. ${ }^{47}$ reported that Nilotinib reduces SARS-CoV-2 infection by $\sim 50 \%$ via interfering with viral cell fusion and replication. In addition, Nilotinib as well as other top ranked molecules (Table S5) were also predicted to bind $\mathrm{RBD}$ in the previous virtual screening studies ${ }^{52,53}$. It is yet unclear if the binding of molecules at RBD interface stabilizes the intermediate states and reduces the population of Up conformation to block ACE2 binding. To shift the conformational equilibrium of S-protein toward the inaccessible Down state for blocking ACE2 and subsequent membrane fusion has been a focus of the recent challenges. Indeed, there have been some reports altering the conformational dynamics of S-protein either via site-specific mutations, disulfide bonds and binding to small molecule ${ }^{14,25,39,54,55,56}$. In this study Nilotinib is only shown as an example, while further studies to identify better molecules targeting the intermediate states would pave the way to hinder viral entry.

The understanding of antibody responses is critical for vaccines and neutralizing antibody developments. The large exposure of $\mathrm{RBD}_{\mathrm{A}}$ in $\mathrm{Up}$ is shown in the current study, which is consistent with several previous studies $^{22,30,57}$. We found that the accessibilities of RBM and the antibody epitopes, including mutational sites, depend sensitively on the RBD conformation, suggesting that each of the Up conformations $\left(1 \mathrm{U}, 1 \mathrm{U}_{\mathrm{O}}\right.$ and $2 \mathrm{U}_{\mathrm{L}}$ ) tends to bind with distinct classes of antibodies. From our inspection, $1 \mathrm{U}_{\mathrm{O}}$, which largely exposes RBM but the antibody epitopes of only Class I and II, is the potentially active conformation for both the ACE2 
binding and evading from the antibody attack. Class I and II epitope regions include K417, E484, and N501, and their mutations could effectively enhance the infection either by enhancing the ACE2 binding (N501Y) ${ }^{5}$ or reducing the antibody binding (K417N or E484K) ${ }^{5}$. Intriguingly, HB analysis shows that K417 and E484 respectively contribute to stabilize the intermediates $\mathrm{I} 2 \mathrm{a} / \mathrm{I} 3 \mathrm{~b}$ and $2 \mathrm{Ua}_{\mathrm{L}}$, likely enhancing the population of oneUp conformations. Note that L452 shows a little accessibility (Fig. 3) in contrast with the reported severe mutational effect to an antibody recognition ${ }^{5}$. The similar observation was also reported previously ${ }^{42}$. A possibility is that L452R mutation affects the structural flexibility of S-protein, which consequently gives rise to the enhanced infection. The last point to mention is that the predicted Up conformations are reasonably aligned with the antibody-bound S-protein Cryo-EM structures (Fig. S13). Hence, the antibody bindings of Sprotein can be explained based on the conformer selection mechanism ${ }^{58}$. The exploration of conformational diversity of S-protein together with binding free-energy calculations followed by docking simulation would provide valuable structural insights with possible antibody bindings $\mathrm{s}^{59}$.

\section{Methods}

\section{gREST_SSCR simulations}

The initial structures for S-protein head regions (residue 28-1135) were prepared based on the Cryo-EM structures PDB:6VXX and PDB:6VYB for the Down and Up states, respectively ${ }^{20} .18 \mathrm{~N}$-glycans and 1 Oglycan were added per protomer as suggested in previous mass-spectrometry experiments and computational models $^{23,60}$. A full list of included glycans is shown in Figure S2. CHARMM-GUI ${ }^{61}$ were used to prepare the final model including the addition of glycans, ions $(0.15 \mathrm{M} \mathrm{NaCl})$ and water molecules. Three gREST_SSCR simulations were performed two from Down in the presence (500 ns) and absence of glycans (150 ns), and one from Up (300 ns). Eight pairs of charged residues per protomer at the RBD interface were selected in the solute region of gREST. The total of number of atoms in solute was 870. All simulations were performed using 16 replicas covering the solute temperature range from 310 to $545 \mathrm{~K}$ while maintaining solvent temperature at $310.15 \mathrm{~K}$. All simulations were performed using the new version of GENESIS MD software that was optimized on Fugaku ${ }^{37,38}$. Further detailed information is given in Supporting Information.

\section{Comparison between Cryo-EM structures and MD simulations using PCA}


We used Cryo-EM structures provided by "Spike protein and spike receptors" in Protein Data Bank (http://www.rcsb.org/covid19, deposited date 2020/02/04-2020/12/11, released by 12/30) in this study. Only structures where the number of residues is greater than 700 and the last residue $>=1122$ for each protomer were selected in the analysis. 373 protomers of 126 structures and 121 trimeric structures from PDB were satisfied with the criteria and used in this study (Table S1). We adopt a method representing the structure with a 9-beads per protomer as used in the previous work of Henderson et al. ${ }^{7,39}$ Unlike their study, 27 beads consisting of three chains are used here. This coarse-grained model consists of 2 beads for RBD, 3 beads for NTD, 1 bead for SD1 and SD2 each, and 2 beads for the S2 region (CD and S2-b) (see Table S2). Principal component analysis (PCA) ${ }^{62}$ was performed using the selected Cryo-EM structures, after converting to the 27 beads model. The PC vectors are calculated upon fitting all the beads. All simulation trajectories were projected onto the PC1 and PC2 vectors and the potential mean forces (PMFs) were calculated for each simulation. The PMF at $310 \mathrm{~K}$ in each gREST_SSCR was obtained using all the trajectories via the Multistate Bennett Acceptance Ratio (MBAR) method ${ }^{40}$. The 373 protomers of the Cryo-EM structures were also used to compare hinge/twist angles.

\section{Pocket Search and Virtual Screening}

The P2Rank software ${ }^{45}$ were used to identify potential druggable pockets in intermediate structures. The cluster centres of $12 \mathrm{a}, 13 \mathrm{a}$ and $13 \mathrm{~b}$ as well as $\mathrm{D} 1_{\text {Sym }}$ and $1 \mathrm{U}_{\mathrm{L}}$ were used for pocket search. All top ranked pockets were investigated. Pockets at the RBD interface were selected for further analysis as they exist in all three intermediates but vanish in Down and Up, representing potential cryptic pockets. To check the druggability of these pockets, all FDA approved drugs were downloaded from ZINC database ${ }^{46}$. This includes 2,115 molecules representing 1,379 drug candidates. Open Babel was used to convert PDB to PDBQT. AutoDockTools-1.5.6 was used to prepare RBD receptor ${ }^{63}$. AutoDock Vina were used to dock all 2115 molecules and perform virtual screening ${ }^{64}$.

\section{Data Availability}

The trajectories are available from the corresponding authors upon reasonable request. Models and PDBs of representative structures in each cluster are available at https://covid.molssi.org/. 


\section{Code Availability}

The source code of GENESIS (https://www.r-ccs.riken.jp/labs/cbrt/) is distributed under the GNU Lesser

General Public License version 3.

\section{References}

1. $\mathrm{Hu}$ B, Guo H, Zhou P, Shi ZL. Characteristics of SARS-CoV-2 and COVID-19. Nat Rev Microbiol 19, 141-154 (2021).

2. Li Y, Tenchov R, Smoot J, Liu C, Watkins S, Zhou Q. A Comprehensive Review of the Global Efforts on COVID-19 Vaccine Development. ACS Cent Sci 7, 512-533 (2021).

3. Venkadapathi J, Govindarajan VK, Sekaran S, Venkatapathy S. A Minireview of the Promising Drugs and Vaccines in Pipeline for the Treatment of COVID-19 and Current Update on Clinical Trials. Front Mol Biosci 8, 637378 (2021).

4. Yurkovetskiy L, et al. Structural and Functional Analysis of the D614G SARS-CoV-2 Spike Protein Variant. Cell 183, 739-751.e8 (2020).

5. Harvey WT, et al. SARS-CoV-2 variants, spike mutations and immune escape. Nat Rev Microbiol 19, 409-424 (2021).

6. Wang P, et al. Antibody resistance of SARS-CoV-2 variants B.1.351 and B.1.1.7. Nature 593, 130135 (2021).

7. Gobeil SM, et al. Effect of natural mutations of SARS-CoV-2 on spike structure, conformation, and antigenicity. Science, 10.1126/science.abi6226, (2021).

8. Prince T, Smith SL, Radford AD, Solomon T, Hughes GL, Patterson EI. SARS-CoV-2 Infections in Animals: Reservoirs for Reverse Zoonosis and Models for Study. Viruses 13, 494 (2021).

9. V'Kovski P, Kratzel A, Steiner S, Stalder H, Thiel V. Coronavirus biology and replication: implications for SARS-CoV-2. Nat Rev Microbiol 19, 155-170 (2021).

10. Finkel Y, et al. The coding capacity of SARS-CoV-2. Nature 589, 125-130 (2021).

11. Ke Z, et al. Structures and distributions of SARS-CoV-2 spike proteins on intact virions. Nature 588, 498-502 (2020).

12. Shang J, et al. Cell entry mechanisms of SARS-CoV-2. Proc Natl Acad Sci U S A 117, 11727-11734 (2020).

13. Wrobel AG, et al. SARS-CoV-2 and bat RaTG13 spike glycoprotein structures inform on virus evolution and furin-cleavage effects. Nat Struct Mol Biol 27, 763-767 (2020).

14. Hsieh CL, et al. Structure-based design of prefusion-stabilized SARS-CoV-2 spikes. Science 369, 1501-1505 (2020).

15. Barnes CO, et al. SARS-CoV-2 neutralizing antibody structures inform therapeutic strategies. Nature 588, 682-687 (2020). 
16. Wrapp D, et al. Cryo-EM structure of the 2019-nCoV spike in the prefusion conformation. Science 367, 1260-1263 (2020).

17. Cai Y, et al. Distinct conformational states of SARS-CoV-2 spike protein. Science 369, 1586-1592 (2020).

18. Wang Q, et al. Structural and Functional Basis of SARS-CoV-2 Entry by Using Human ACE2. Cell 181, 894-904.e9 (2020).

19. Yan R, Zhang Y, Li Y, Xia L, Guo Y, Zhou Q. Structural basis for the recognition of SARS-CoV-2 by full-length human ACE2. Science 367, 1444-1448 (2020).

20. Walls AC, Park YJ, Tortorici MA, Wall A, McGuire AT, Veesler D. Structure, Function, and Antigenicity of the SARS-CoV-2 Spike Glycoprotein. Cell 181, 281-292.e6 (2020).

21. Fan X, Cao D, Kong L, Zhang X. Cryo-EM analysis of the post-fusion structure of the SARS-CoV spike glycoprotein. Nat Commun 11, 3618 (2020).

22. Grant OC, Montgomery D, Ito K, Woods RJ. Analysis of the SARS-CoV-2 spike protein glycan shield reveals implications for immune recognition. Sci Rep 10, 14991 (2020).

23. Watanabe Y, Allen JD, Wrapp D, McLellan JS, Crispin M. Site-specific glycan analysis of the SARSCoV-2 spike. Science 369, 330-333 (2020).

24. Barnes CO, et al. Structures of Human Antibodies Bound to SARS-CoV-2 Spike Reveal Common Epitopes and Recurrent Features of Antibodies. Cell 182, 828-842.e16 (2020).

25. Juraszek J, et al. Stabilizing the closed SARS-CoV-2 spike trimer. Nat Commun 12, 244 (2021).

26. Xu C, et al. Conformational dynamics of SARS-CoV-2 trimeric spike glycoprotein in complex with receptor ACE2 revealed by cryo-EM. Sci Adv 7, eabe5575 (2021).

27. Lu M, et al. Real-Time Conformational Dynamics of SARS-CoV-2 Spikes on Virus Particles. Cell Host Microbe 28, 880-891.e8 (2020).

28. Turonova B, et al. In situ structural analysis of SARS-CoV-2 spike reveals flexibility mediated by three hinges. Science 370, 203-208 (2020).

29. Choi YK, et al. Structure, Dynamics, Receptor Binding, and Antibody Binding of the Fully Glycosylated Full-Length SARS-CoV-2 Spike Protein in a Viral Membrane. J Chem Theory Comput 17, 2479-2487 (2021).

30. Casalino L, et al. Beyond Shielding: The Roles of Glycans in the SARS-CoV-2 Spike Protein. ACS Cent Sci 6, 1722-1734 (2020).

31. Mori T, Jung J, Kobayashi C, Dokainish HM, Re S, Sugita Y. Elucidation of interactions regulating conformational stability and dynamics of SARS-CoV-2 S-protein. Biophys $J$ 120, 1060-1071 (2021).

32. Gur M, Taka E, Yilmaz SZ, Kilinc C, Aktas U, Golcuk M. Conformational transition of SARS-CoV2 spike glycoprotein between its closed and open states. J Chem Phys 153, 075101 (2020).

33. Fallon L, et al. Free Energy Landscapes from SARS-CoV-2 Spike Glycoprotein Simulations Suggest that RBD Opening Can Be Modulated via Interactions in an Allosteric Pocket. J Am Chem Soc 143, 11349-11360 (2021). 
34. Zimmerman MI, et al. SARS-CoV-2 simulations go exascale to predict dramatic spike opening and cryptic pockets across the proteome. Nat Chem 13, 651-659 (2021).

35. Sztain $\mathrm{T}$, et al. A glycan gate controls opening of the SARS-CoV-2 spike protein. bioRxiv, 10.1101/2021.02.15.431212, (2021).

36. Dokainish HM, Sugita Y. Exploring Large Domain Motions in Proteins Using Atomistic Molecular Dynamics with Enhanced Conformational Sampling. Int J Mol Sci 22, 270 (2020).

37. Kobayashi C, et al. GENESIS 1.1: A hybrid-parallel molecular dynamics simulator with enhanced sampling algorithms on multiple computational platforms. J Comput Chem 38, 2193-2206 (2017).

38. Jung J, et al. New parallel computing algorithm of molecular dynamics for extremely huge scale biological systems. J Comput Chem 42, 231-241 (2021).

39. Henderson R, et al. Controlling the SARS-CoV-2 spike glycoprotein conformation. Nat Struct Mol Biol 27, 925-933 (2020).

40. Shirts MR, Chodera JD. Statistically optimal analysis of samples from multiple equilibrium states. $J$ Chem Phys 129, 124105 (2008).

41. Zhang C, et al. Development and structural basis of a two-MAb cocktail for treating SARS-CoV-2 infections. Nat Commun 12, 264 (2021).

42. Valdes-Balbin Y, et al. Molecular Aspects Concerning the Use of the SARS-CoV-2 Receptor Binding Domain as a Target for Preventive Vaccines. ACS Cent Sci 7, 757-767 (2021).

43. Pinto D, et al. Cross-neutralization of SARS-CoV-2 by a human monoclonal SARS-CoV antibody. Nature 583, 290-295 (2020).

44. Brotzakis ZF, Lohr T, Vendruscolo M. Determination of intermediate state structures in the opening pathway of SARS-CoV-2 spike using cryo-electron microscopy. Chem Sci 12, 9168-9175 (2021).

45. Krivak R, Hoksza D. P2Rank: machine learning based tool for rapid and accurate prediction of ligand binding sites from protein structure. J Cheminform 10,39 (2018).

46. Irwin JJ, Shoichet BK. ZINC--a free database of commercially available compounds for virtual screening. J Chem Inf Model 45, 177-182 (2005).

47. Cagno V, Magliocco G, Tapparel C, Daali Y. The tyrosine kinase inhibitor nilotinib inhibits SARSCoV-2 in vitro. Basic Clin Pharmacol Toxicol 128, 621-624 (2021).

48. Henderson R, et al. Glycans on the SARS-CoV-2 Spike Control the Receptor Binding Domain Conformation. bioRxiv, 10.1101/2020.06.26.173765, (2020).

49. Li Q, et al. The Impact of Mutations in SARS-CoV-2 Spike on Viral Infectivity and Antigenicity. Cell 182, 1284-1294.e9 (2020).

50. Han N, et al. Identification of SARS-CoV-2-induced pathways reveals drug repurposing strategies. Sci $A d v$ 7, eabh3032 (2021).

51. Bakowski MA, et al. Drug repurposing screens identify chemical entities for the development of COVID-19 interventions. Nat Commun 12, 3309 (2021). 
52. Deganutti G, Prischi F, Reynolds CA. Supervised molecular dynamics for exploring the druggability of the SARS-CoV-2 spike protein. J Comput Aided Mol Des 35, 195-207 (2021).

53. Murugan NA, Kumar S, Jeyakanthan J, Srivastava V. Searching for target-specific and multi-targeting organics for Covid-19 in the Drugbank database with a double scoring approach. Sci Rep 10, 19125 (2020).

54. Edwards RJ, et al. Cold sensitivity of the SARS-CoV-2 spike ectodomain. Nat Struct Mol Biol 28, 128-131 (2021).

55. McCallum M, Walls AC, Bowen JE, Corti D, Veesler D. Structure-guided covalent stabilization of coronavirus spike glycoprotein trimers in the closed conformation. Nat Struct Mol Biol 27, 942-949 (2020).

56. Toelzer C, et al. Free fatty acid binding pocket in the locked structure of SARS-CoV-2 spike protein. Science 370, 725-730 (2020).

57. Sikora M, von Bulow S, Blanc FEC, Gecht M, Covino R, Hummer G. Computational epitope map of SARS-CoV-2 spike protein. PLoS Comput Biol 17, e1008790 (2021).

58. Csermely P, Palotai R, Nussinov R. Induced fit, conformational selection and independent dynamic segments: an extended view of binding events. Trends Biochem Sci 35, 539-546 (2010).

59. Ren W, Dokainish HM, Shinobu A, Oshima H, Sugita Y. Unraveling the Coupling between Conformational Changes and Ligand Binding in Ribose Binding Protein Using Multiscale Molecular Dynamics and Free-Energy Calculations. J Phys Chem B 125, 2898-2909 (2021).

60. Woo H, et al. Developing a Fully Glycosylated Full-Length SARS-CoV-2 Spike Protein Model in a Viral Membrane. J Phys Chem B 124, 7128-7137 (2020).

61. Lee J, et al. CHARMM-GUI Input Generator for NAMD, GROMACS, AMBER, OpenMM, and CHARMM/OpenMM Simulations Using the CHARMM36 Additive Force Field. J Chem Theory Comput 12, 405-413 (2016).

62. Yang LW, Eyal E, Bahar I, Kitao A. Principal component analysis of native ensembles of biomolecular structures (PCA_NEST): insights into functional dynamics. Bioinformatics 25, 606-614 (2009).

63. Morris GM, et al. AutoDock4 and AutoDockTools4: Automated docking with selective receptor flexibility. J Comput Chem 30, 2785-2791 (2009).

64. Trott O, Olson AJ. AutoDock Vina: improving the speed and accuracy of docking with a new scoring function, efficient optimization, and multithreading. J Comput Chem 31, 455-461 (2010). 


\section{Acknowledgements (optional)}

This research used computational resources of the supercomputer Fugaku (The evaluation environment in the trial phase) provided by the RIKEN Center for Computational Science. The results obtained on the evaluation environment in the trial phase do not guarantee the performance, power and other attributes of the supercomputer Fugaku at the start of its public use operation. The computer resources of Oakforest-PACS were also provided through HPCI System Research project. (Project ID: hp200153, hp200028). The research was supported in part by MEXT as "FLAGSHIP 2020 project", "Priority Issue on Post-K computer" (Building Innovative Drug Discovery Infrastructure Through Functional Control of Biomolecular Systems), "Program for Promoting Researches on the Supercomputer Fugaku" (Biomolecular dynamics in a living cel1/MD-driven Precision Medicine), and MEXT/KAKENHI (Grant Numbers 19H05645 (to YS), 20K15737 (to H.M.D.), 19K12229 (to SR), and 19K06532 (to TM)) and by RIKEN Pioneering Research Projects (Dynamic Structural Biology/Glycolipidologue Initiative/Biology of Intracellular Environments) (to YS).

\section{Ethics declarations}

\section{Competing interests}

The authors declare no competing interests.

\section{Supplementary Information}

Supporting information is available for this paper. 
a

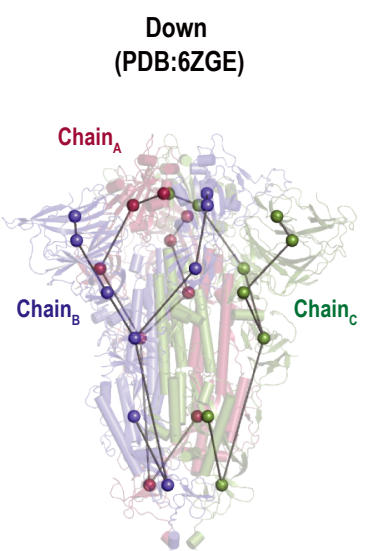

b

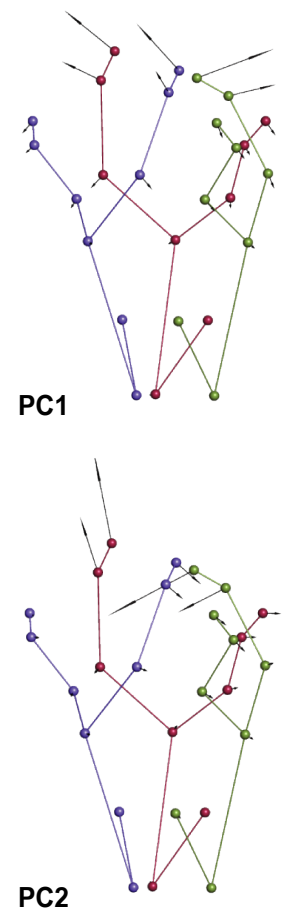

1Up

(PDB:6XKL)

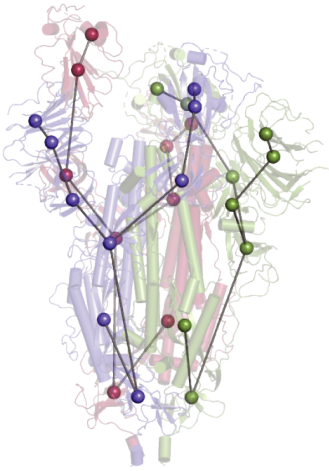

$2 U p$

(PDB:7K8U)

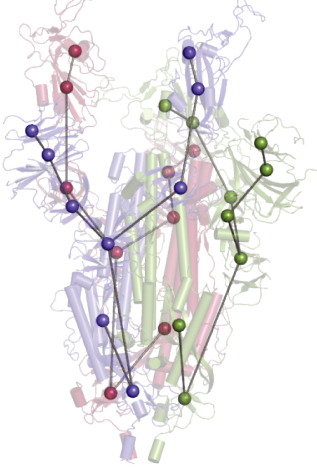

3 Up

(PDB:6XCN)

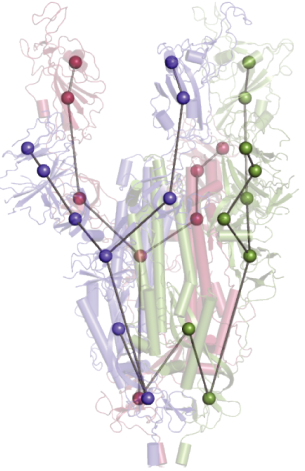

C

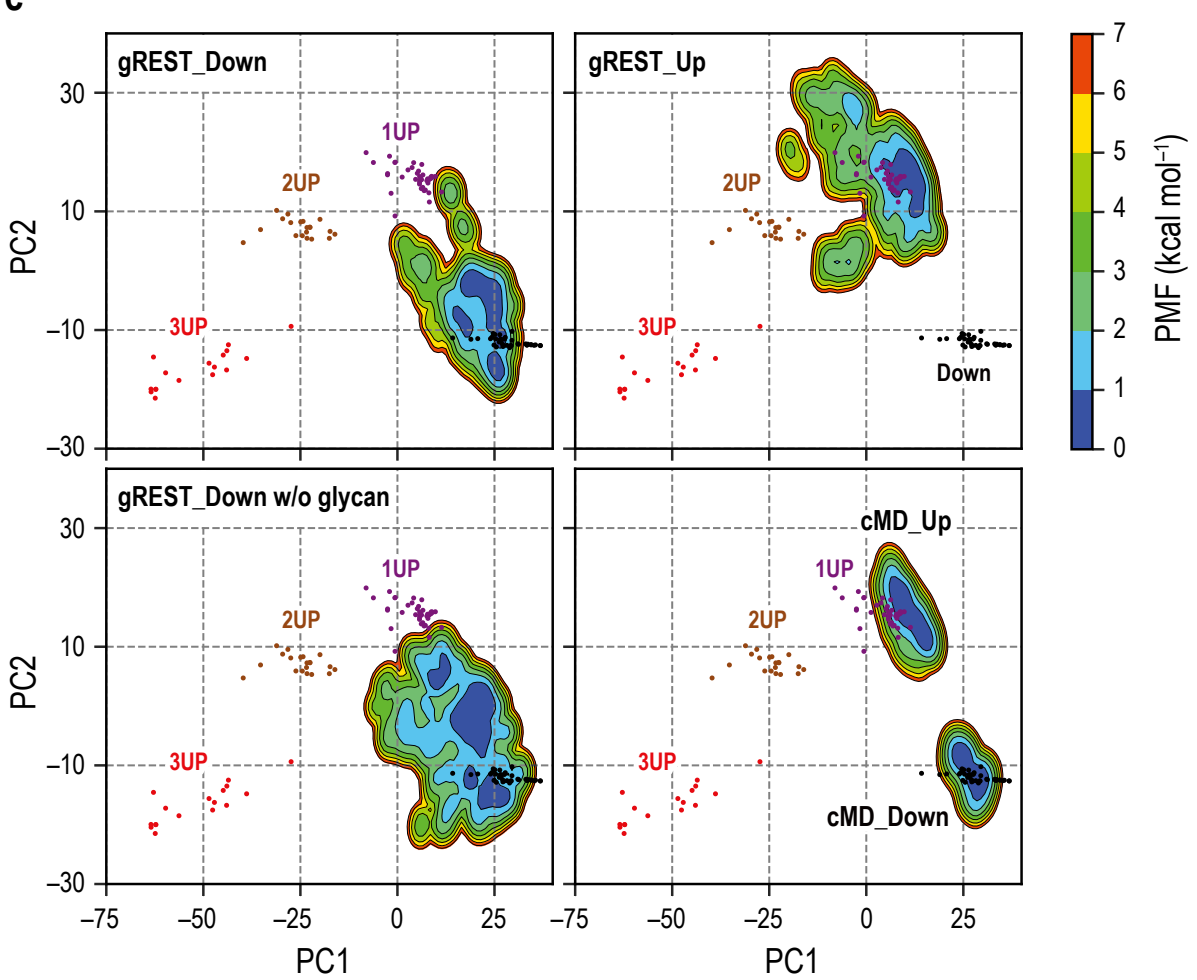

Figure 1. Comparisons of S-protein structures in MD simulations with Cryo-EM structures. a) 27 coarsegrained beads representations of four representative Cryo-EM structures: Down (PDB ID: 6ZGE), 1Up (6XKL), 2Up (7K8U), and 3Up (6XCN). Chain A, B, and C in S-protein are shown in red, blue, and green, respectively. b) The lowest two modes (PC1 and PC2) in principal component analysis of the 27 beads model of 126 Cryo-EM structures. PC1 and PC2 respectively represent a symmetric and an antisymmetric Down to Up motion of the receptor binding domains. The vectors are magnified 100 times for clarity. c) Free energy landscapes at $310 \mathrm{~K}$ along the PC1 and PC2 obtained from the simulations: (top) gREST_SSCR simulations with glycans starting from Down (500 ns) and Up (300 ns), (bottom left) gREST_SSCR simulation without glycans from Down (150 ns), (bottom right) conventional MD simulations with glycans starting from either Down and Up. The positions of Cryo-EM structures are also shown for comparison. Wherein, Down, 1Up, $2 \mathrm{Up}$ and $3 \mathrm{Up}$ conformations are shown in black, purple, brown and red dots, respectively. 
a

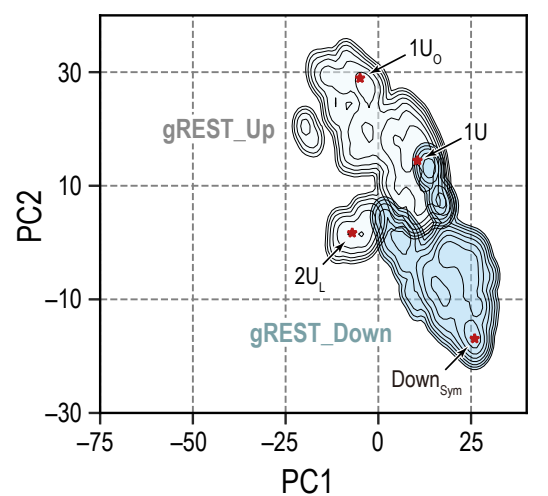

b

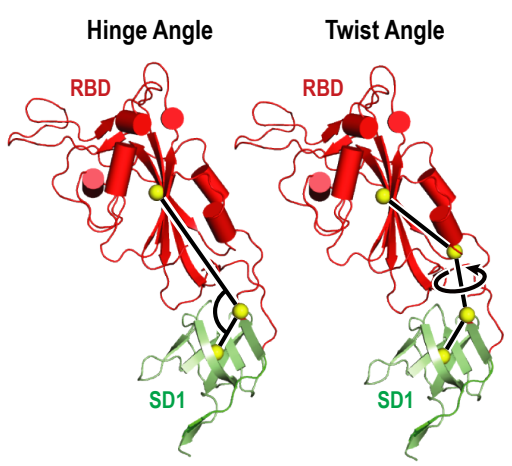

C

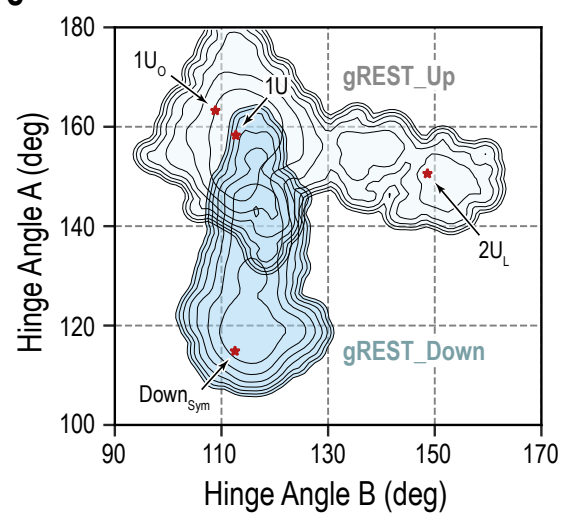

d Down $_{\text {sym }}$ vs. PDB:6ZGE

1U vs. PDB:6XKL

$1 \mathrm{U}_{0}$ vs. PDB:7DCC

$2 \mathrm{U}_{\mathrm{L}}$ vs. $\mathrm{PDB}: 6 \mathrm{X} 2 \mathrm{~B}$
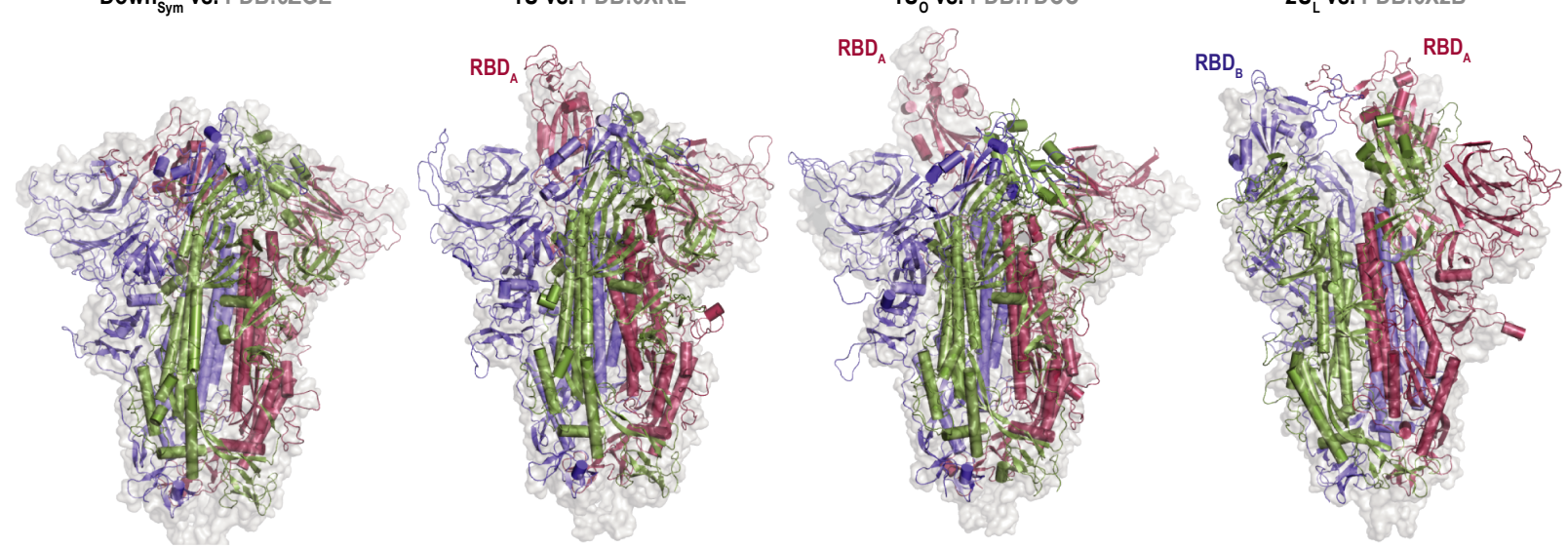

Figure 2. Representative RBD conformations from MD simulations. a) An overlay of the two free energy landscapes at $310 \mathrm{~K}$ along the PC1 and PC2 obtained from gREST_Down (light blue) and gREST_Up (light cyan) simulations. The red dots represent the positions of four representative RBD conformations: symmetric Down $\left(\right.$ Down $\left._{\text {Sym }}\right), 1 R B D$ Up (1U and $\left.1 U_{O}\right)$ and 2RBDs Up like $\left(2 U_{L}\right)$ conformations. b) Definitions of the hinge and twist angles representing the RBD conformations. The hinge angle is determined by three center of masses (COMs, yellow spheres) of the core and top residues of SD1 (green, C $\alpha$ atoms only) and the core residues of RBD (red, $\mathrm{C} \alpha$ atoms only). The twist angle is determined by the aforementioned COMs with an extra COMs of the bottom residues of RBD. c) An overlay of two free energy landscapes at $310 \mathrm{~K}$ along the hinge angles in $\mathrm{RBD}_{\mathrm{A}}$ and $\mathrm{RBD}_{\mathrm{B}}$ obtained from gREST_Down (light blue) and gREST_Up (light cyan) simulations. d) Four representative conformations from MD simulations (cartoon representation) in comparison with Cryo-EM structures (light grey surface). Chain A, B, and C in S-protein are shown in red, blue, and green, respectively. 

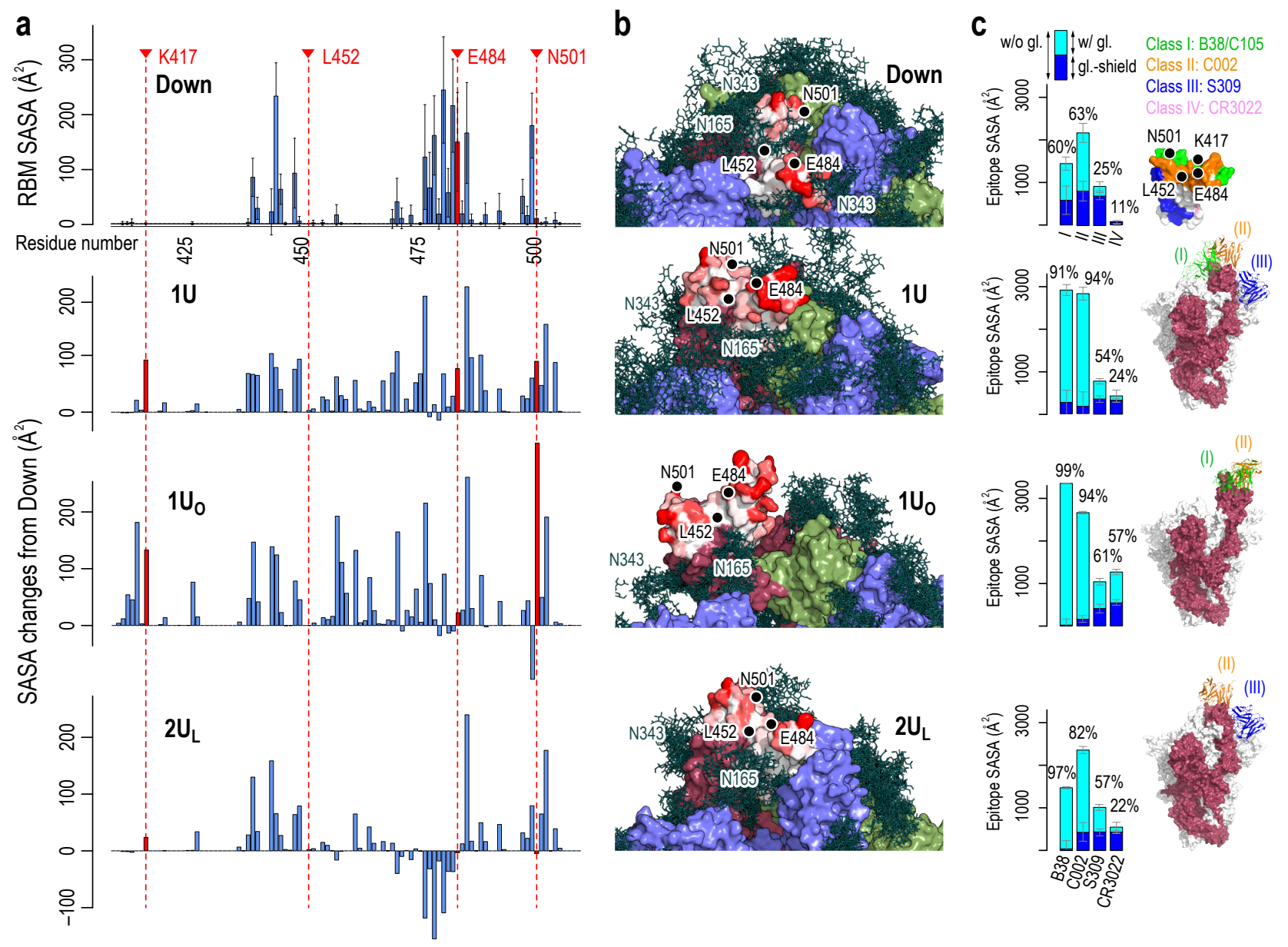

Figure 3. Accessibility of receptor binding motif (RBM). a) Per-residue solvent accessible surface area (SASA) values of the receptor binding motif (RBM, residues 410 to 510) in Down conformation (top) and their changes in Up conformations (bottom three). SASA values were calculated using the probe radius of 7.2 $\AA$. Four mutational residues, K417, L452, E484, and N501, are highlighted in red. b) The surface representation of RBM SASA (white to red for 0 to $260 \AA^{2}$, the maximum value in Down, the values higher than this are truncated for consistent color scheme). Chain A, B, and C in the protein are shown in red, blue, and green surfaces, respectively, while a collection of glycans from 10 snapshots are shown in stick representation. c) Epitope SASA and glycan shielding of four types of neutralized antibodies, B38 (Class I), C002 (Class II), S309 (Class III), and CR3022 (Class IV). Sum of SASA with glycans (cyan) and the glycanshield (blue) gives SASA without glycans. The ratio of the SASA with glycan over that without glycans is shown. The right most column show the putative interaction models with three classes of antibodies: Class III (S309, PDBID: 6WPT, blue), Class II (C002, PDBID: 7K8T, orange), and Class I (C105, PDBID: 6XCM, green). 


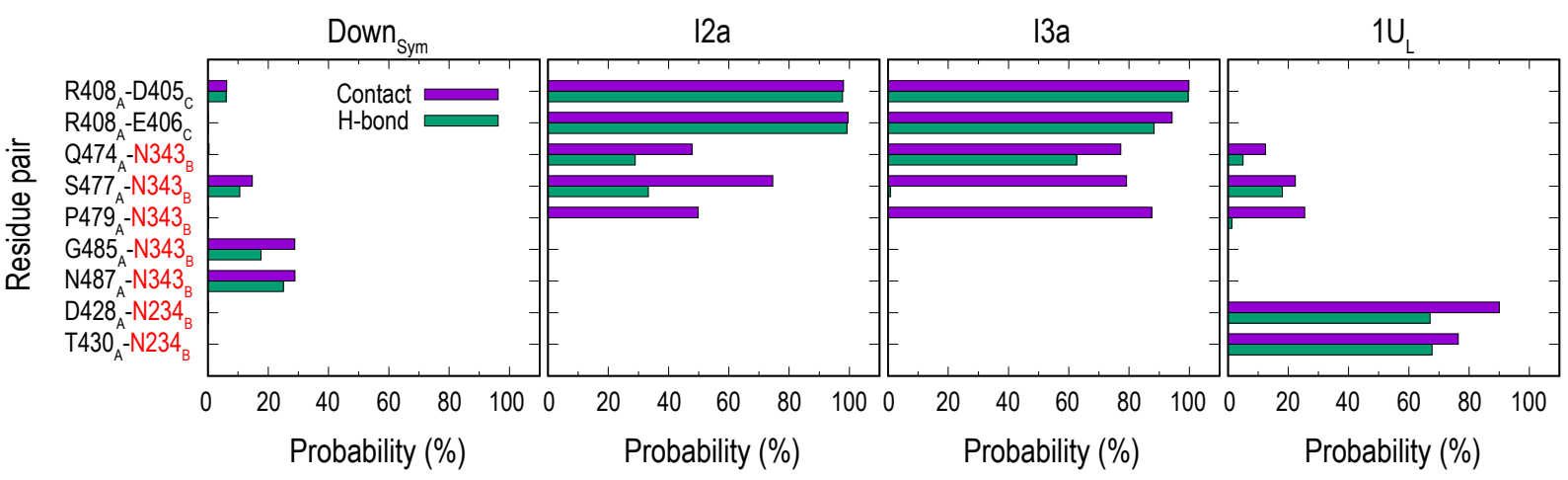

b
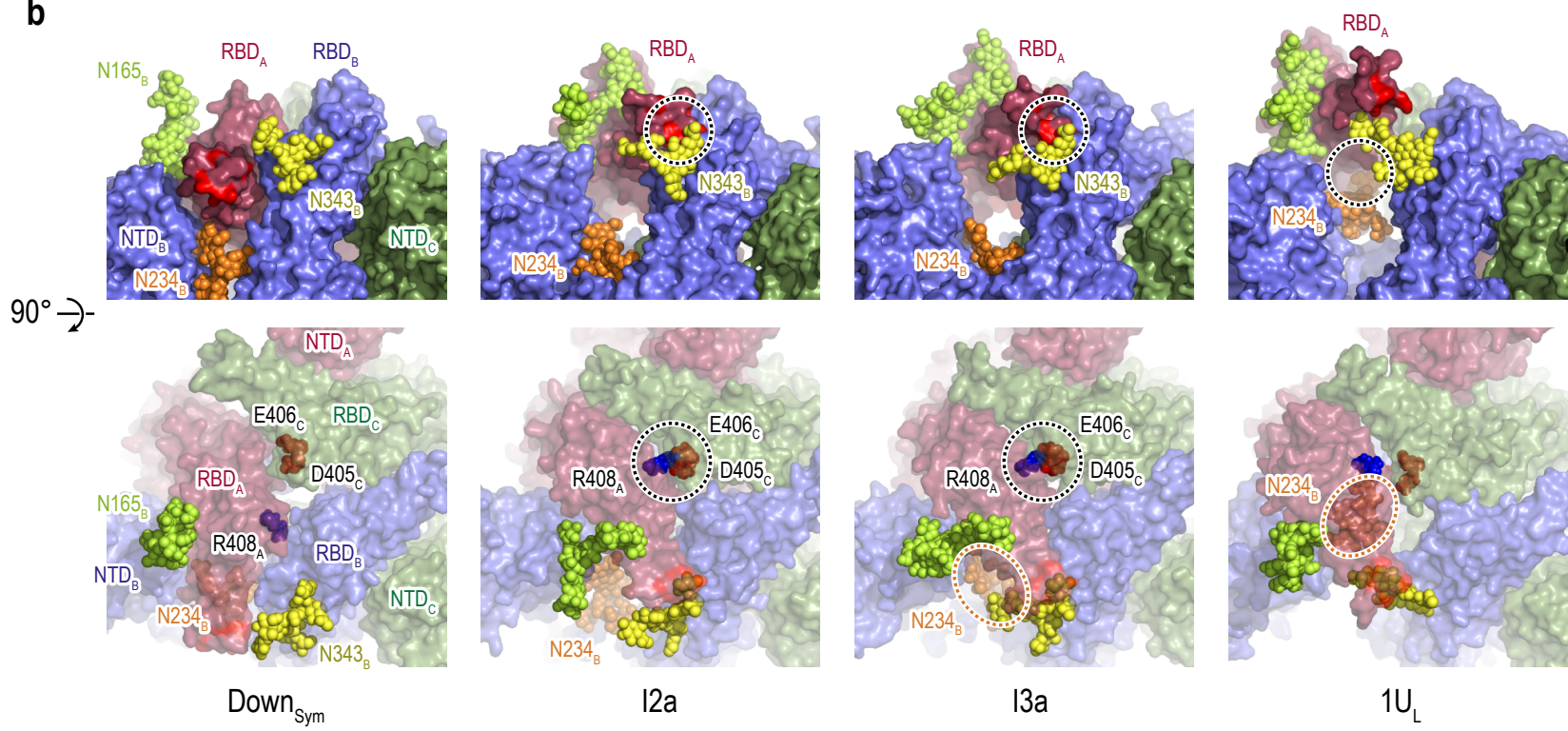

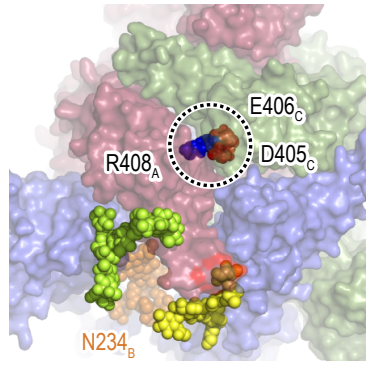

$12 \mathrm{a}$

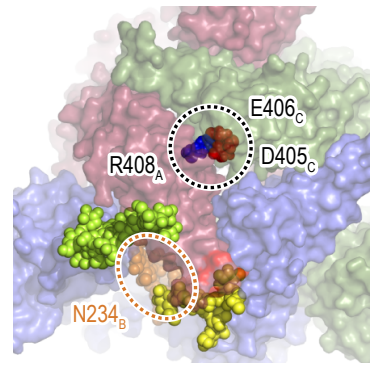

$13 a$

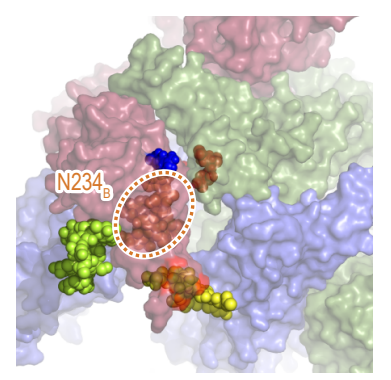

$1 U_{L}$

Figure 4. Protein-protein and protein-glycan interactions critical for Down-to-Up transition. a) Probability of finding the hydrogen bond (green) and contact (purple) pairs between protein residues or protein-glycans that markedly change along the transition pathway $\left(\mathrm{D}_{\mathrm{Sym}}, \mathrm{I} 2 \mathrm{a}, \mathrm{I3a}\right.$ and $1 \mathrm{U}_{\mathrm{L}}$ ). All hydrogen bond (probability of finding of $>50 \%$ ) and contact pairs (probability of finding of $>70 \%$ ) are shown in figure S19 and S20, respectively. b) Typical snapshots of the protein-protein and protein-glycan interactions along the transition pathway. Chain A, B, and C in the protein are shown in red, blue, and green surfaces, respectively. Glycans at N165, N234 and N343 are shown with spheres in lime, orange and yellow color, respectively. The transient $\mathrm{N} 343_{\mathrm{B}}-\mathrm{RBD}_{\mathrm{A}}$ contact are highlighted in red surface (top). The salt-bridges formed by R408 $\mathrm{A}_{\mathrm{A}}$ (blue), E406 (brown) and D405 (red) are also highlighted with black dashed circles (top and bottom), while the location of $\mathrm{N} 234$ glycan is highlighted with orange dashed circles. 
a

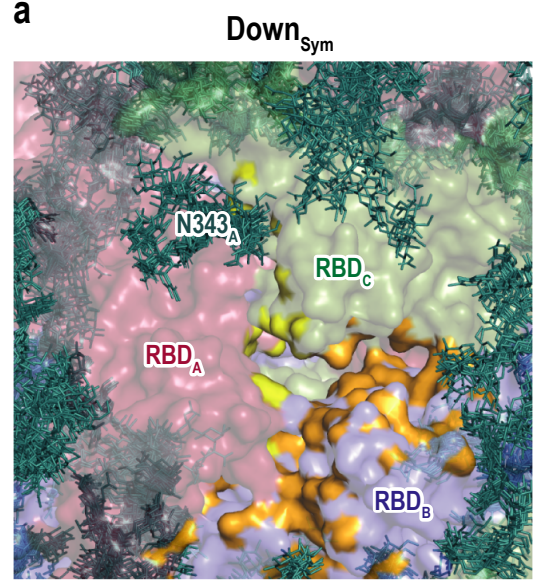

b

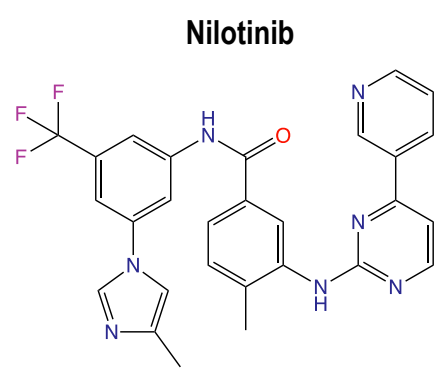

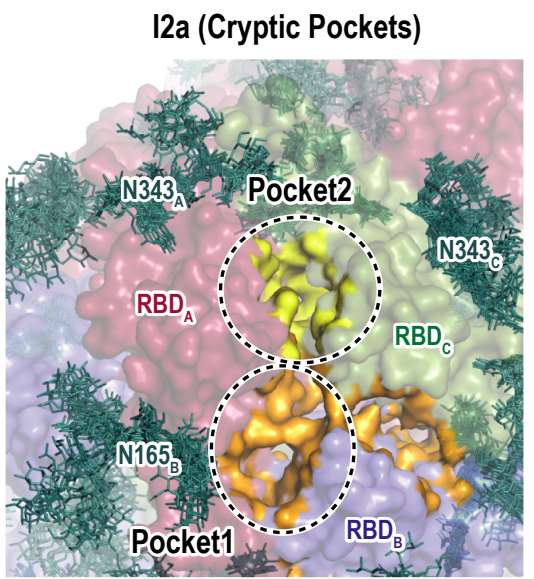

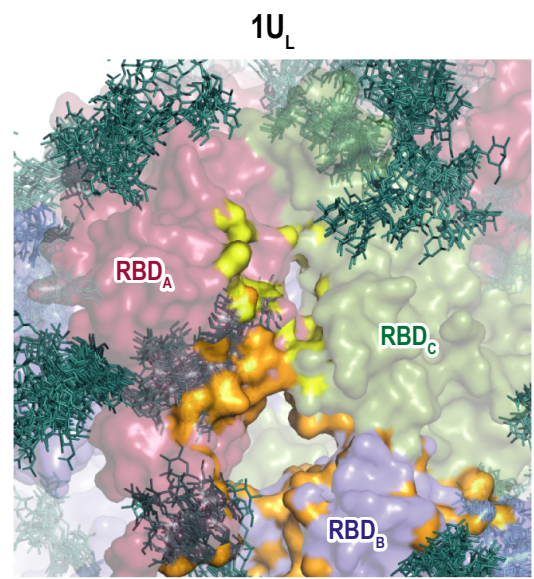

C

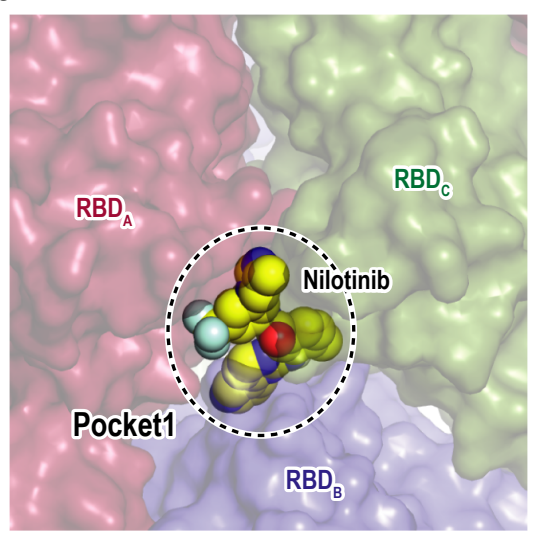

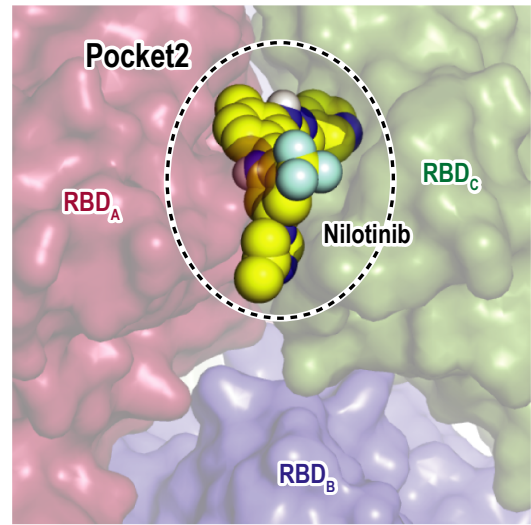

Figure 5. Druggable cryptic pockets. a) Snapshots of RBD interface in Down symmetric (Down $n_{\text {Sym }}$ ), Intermediate $2 \mathrm{a}(\mathrm{I} 2 \mathrm{a})$ and $1 \mathrm{Up}$ like $\left(1 \mathrm{U}_{\mathrm{L}}\right)$ conformations. Chain $\mathrm{A}, \mathrm{B}$, and $\mathrm{C}$ in the protein are shown in red, blue, and green surfaces, respectively, while a collection of glycans from 10 snapshots are shown in stick representation. The cryptic pockets predicted for I2a using P2Rank software are shown in orange (Pocket1) and yellow (Pocket2), respectively. These pockets disappear in both Down $n_{\text {Sym }}$ and $1 U_{L}$. b) Chemical structure of Nilotinib and c) the docked poses (top and third ranked) to two cryptic pockets in I2a by Autodock Vina. The pockets are highlighted with black dashed circles and the nilotinib is shown in sphere representation in yellow. 